\title{
Structural Equation Modeling for the Effect of Main Factors on Abortion Issue
}

\author{
Aras Jalal Mhamad \\ Statistic \& Informatics Department, College of Administration \& Economics, University of Sulaimani, \\ Sulaimani,Kurdistan Region, Iraq \\ Email: aras.mhamd@univsul.edu.iq

\section{Renas Abubaker Ahmed} \\ Statistic \& Informatics Department, College of Administration \& Economics, University of \\ Sulaimani, Sulaimani,Kurdistan Region, Iraq \\ Email: renas.ahmed@univsul.edu.iq
}

\begin{abstract}
:
Based on medical exchange and medical information processing theories with statistical tools, our study proposes and tests a research model that investigates main factors behind abortion issue. Data were collected from the survey of Maternity hospital in Sulaimani, Kurdistan-Iraq. Structural Equation Modelling (SEM) is a powerful technique as it estimates the causal relationship between more than one dependent variable and many independent variables, which is ability to incorporate quantitative and qualitative data, and it shows how all latent variables are related to each other. The dependent latent variable in SEM which have one-way arrows pointing to them is called endogenous variable while others are exogenous variables. The structural equation modeling results reveal is underlying mechanism through which statistical tools, as relationship between factors; previous disease information, food and drug information, patient address, mother's information, abortion information, which are caused abortion problem. Simply stated, the empirical data support the study hypothesis and the research model we have proposed is viable. The data of the study were obtained from a survey of Maternity hospital in Sulaimani, Kurdistan-Iraq, which is in close contact with patients for long periods, and it is number one area for pregnant women to obtain information about the abortion issue. The results shows arrangement about factors effectiveness as mentioned at section five of the study. This gives the conclusion that abortion problem must be more concern than the other pregnancy problem.
\end{abstract}

Keywords: - Structural Equation Model, Abortion Situation Analysis, Data Analysis 


\subsection{Introduction:}

The abortion issue is one of the most important medical problems, because of abortion is the major and most dangerous phenomenon, which causes loss of life, each year in the world 208 million women estimated to become pregnant, among this amount 59\% (123 million) have a planned (intended) pregnancies, that results to birth or abortion or a birth of a dead fetus ${ }^{[30]}, 41 \%$ (85 million) of pregnancies are unplanned, induced abortion decreased from 35 per 1000 in 1995 to1995 to 26 per 1000 in 2008 for women aged 15-44 years, however the coverage of unsafe abortion remain fixed since 2000 at about 14 per 1000 for the same age ${ }^{[29]}$. The entire number of unsafe abortions was rated at about 20 million in 2003 and 22 million in 2008. Treatment of complications of unsafe abortion is confusing for it is many costs, especially in poor countries, so the safe abortion is considered to be a cost saving ${ }^{[32]}$. According to a recent study the cost of treating a minor complication from unsafe abortion is estimated to be US\$23 million per year, US\$ 6 billion are spend to treat infertility after abortion ${ }^{[32]}$. So to solve this issue several statistical tools exist such as structural Equation Models (SEMs) which are widely used, e.g., in biomedical, educational, behavioral, psychological, and social sciences. Many methods have been developed to fit SEMs ${ }^{[2]}$. Structural equation models (SEMs) are often formulated using a pre-defined parametric structural equation are often used to formulate Structural equation models (SEM), while the formulation of the structural equation remain unknown in many applications, this misspecification may give rise to uncertain statistical inference ${ }^{[34]}$. So, there is a requirement to improve a general SEM in which potential variable model is exploratory ${ }^{[33]}$. A framework by mean of model compare with some standard such as Akaike Information Criterion, the Bayesian Information Criterion or the Deviance Information Criterion are used by some anthers to choose a certain form of the latent variable model in an SEM ${ }^{[23]}$.

\subsection{Objective of the study:}

Our study develops and proposes a research model that examines the relationship between factors which are caused abortion, generally, the study tests the relation and impacts of previous disease information, food and drug information, patient address, and mother's information as the indicators (factors) on occurring abortion issue.

\section{Theoretical and literature review}

\subsection{Methods of abortion :}

Many steps are taken to provide abortion care, the first is to make sure that the women is, and to estimate the period of the pregnancy, and to prove that the pregnancy is intrauterine. Even though, the abortion is properly performed, the risks connected to induce abortion increase depending on the duration of pregnancy ${ }^{[5]}$. Three methods are used for the first-trimester abortion; the first one is manual or electric vacuum aspiration for up to $12-14$ weeks pregnancies. the second method is the medical method abortion that is oral mifepristone and a single dose of misoprostol for up to 63 days pregnancies, the third method is medical method as well for over 63 days pregnancies. For more than 12-14 weeks of pregnancies dilatation and curettage (D\&C) is an old method of surgical abortion and it should be displaced by vacuum aspiration and medical methods, for gestational age more than 12-14 weeks of pregnancies, those methods are showed; the first method is dilatation and evacuation (D\&E) by using vacuum aspiration and forceps or mifepristone followed by period doses of misoprostol and if mifepristone is not available misoprostol is used alone. The second method is for all women with over 12-14 weeks pregnancy that is cervical preparation before surgical abortion is recommended for pain handling for medical and surgical abortions, the third method is worked to provide medication to women who need it. 
Standard precautions should be used to control infection, to reduce the risk of blood-borne transmission and that is the fourth method ${ }^{[31]}$.

\section{2 literature review:}

A general review of the empirical studies on abortion problem reveals that there is little empirical evidence pertaining to the use meaningful statistical tools such as structural equation model to investigating factors which are caused occurring abortion issue.

Jiang et al. (2017) ${ }^{[20]}$ study in Uruguay reported that the women were divided into either the incomplete medical abortion group $(n=34)$ or the complete medical abortion group $(n=29)$, compared with the complete abortion group, there was a marked decrease in the expression of insulin-like growth factor-1 and a significant increase in the expression of vascular endothelial growth factor $(\mathrm{p}<0.05)$ in the incomplete abortion group. There was no significant difference in mRNA expression between the incomplete and complete abortion groups. Leonel Briozzo, (2016) ${ }^{[25]}$ found that changes in public policies and expansion of the risk reduction model coincided with changes in the social perception of abortion and a decrease in maternal mortality and abortion rates, probably due to a set of public policies that led to the decriminalization of abortion in 2012. Oliver-Williams et al. (2015) ${ }^{[27]}$ discussed that overall, 196040 women were included, compared with white Europeans, the odds of a previous spontaneous abortion were increased in black African and black Caribbean women, the strength of the association with black African race increased with age, and the association with South Asian race increased with age and body mass index, spontaneous abortion was associated with preterm birth in all races, but was strongest in black African women. Mamboleo (2012) ${ }^{[26]}$ documented about unwanted pregnancy and induced abortion, the findings indicate to the prevalence of unwanted pregnancy and induced abortion were high, female youths who were single, unmarried and students were found to have the high likelihood of having the unwanted pregnancy and induced abortion. On the other hand, Ahmed et al. (1996) ${ }^{[3]}$ recommended that to safe and controlling abortion problem should focus more on the mothers' age, number of living children and prior contraception as the result of their study.

\subsection{Confirmatory Factor Analysis (CFA):}

To test hypotheses about the relationships among observed and latent variables, the structural equation modeling is a statistical approach. The latent variables cannot be measured directly is used ${ }^{[16]}$. Researchers must explain the latent variable in terms of observed variables cannot be calculated instantly. SEM is also an approach that takes a probative (hypothesis testing) method to the analysis of a theory relating to some event. A measurement model and a structural model are two parts of Structural Equation Models, to deal with the relation between measured variables and latent variables the measurement model is used, for the relationships between latent variables only the structural model is used. Measured variables are signalized by rectangles or squares while latent variables are signalized by circles

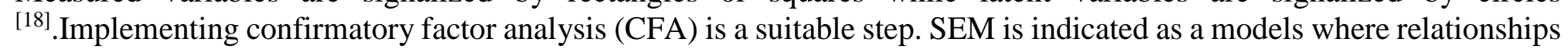
are hypothesized to occur between latent variables. While the CFA define whether the hypothesized framework provides a perfect fit to the data that mean the relationship between the observed variables and latent variable structure occur ${ }^{[11]}$, so the (CFA) is considered to be a special case of (SEM). Can be provide evidence that the variables are fairly ranged with proper latent variables within the generic form being measured by using the CAF. The CFA can be run by PROC CALIS (Covariance Analysis of Linear Structural Equations). 


\subsection{Assessing CAF Fit Statistics and Fit Indices:}

When we work on CFA, various relevance statistics are used to decide whether the model provides an appropriate fit for the data. To show the difference between expected and observed covariance matrices the chi-square test is used, if the value of chi-square is close to zero and p-value is greater than 0.05 then there is a small amount of difference between the observed and expected covariance matrices, and it is one indicator of good fit ${ }^{[22]}$.

Also, there are two indices which are used frequently are Bentler's comparative fit index (CFI) and the root mean square error of approximation (RMSEA), The CFI is a comparison of the fit of the researcher's model to the fit of a null model. The null model is highly constrained and unrealistic. More specifically, the model parameters are constrained such that all covariances among measured variables are equal to zero, CFI is defined as:

$$
C F I_{\text {pop }}=\frac{\lambda_{\text {null model }}-\lambda_{\text {researcher's model }}}{\lambda_{\text {null model }}}
$$

where $\lambda$ is a non-central parameter that is an indicator of lack of fit of the model to a population covariance matrix. If we get a proper model the value of $\lambda$ will be zero and becomes greater if the model is miss-specified, in most applications of SEM the $\lambda_{\text {null model }}$ would be large because we expect it's null model to be miss-specified, so we expect to that the,$\lambda_{\text {researcher's model }}$ to be smaller; consequently a high CFI value is model is better than the null model that done uncorrelated measured variables. A sample estimated of CFI in the of the $C F I_{\text {pop }}$ we can substitute $\mathrm{T}$ - df for $\lambda$ to obtain a sample estimate of CFI:

$$
C F I=\frac{\left(T_{\text {null model }}-d f_{\text {null model }}\right)-\left(T_{\text {researcher's model }}-d f_{\text {researcher's model }}\right)}{\left(T_{\text {null model }}-d f_{\text {null model }}\right)} \ldots \ldots
$$

CFI values range from zero to one with a larger value indicating better model fit ${ }^{[10]}$. The RMSEA is a fit index that assesses lack of fit, but does not use the unrealistic comparison of a null model. The sample estimate is also a function of $\mathrm{T}$ and df:

$$
R M S E A=\sqrt{\frac{T_{\text {researcher's model }} /(N-1)}{d f_{\text {researcher's model }}}-\frac{1}{(N-1)}}
$$

To the extent that the model fits [i.e., small $T_{\text {researcher's model }} /(N-1)$ ] and the model involves estimating few model parameters (large $d f_{\text {researcher's model }}$ ), RMSEA should approach zero. The Root Mean Square Error of Approximation (RMSEA) is related to the residuals in the model. RMSEA values range from zero to one with a smaller RMSEA value indicating better model fit ${ }^{[19]}$.

The goodness of fit index (GFI) is the other indicators that are commonly used to measure model fit ${ }^{[6]}$, was devised by (Jöreskog and Sörbom) ${ }^{[21]}$, which is calculated as:

$$
G F I=1-\frac{\widehat{F}}{\widehat{F}_{b}}
$$

where $\hat{F}$ is the minimum value of the discrepancy function and $\hat{F}_{b}$ is obtained by evaluating $\mathrm{F}$, for this indicator, a larger value specify better model fit. Bollen's incremental fit index (IFI) is the other indicators were calculated by ${ }^{[8]}$ :

$$
I F I=\Delta_{2}=\frac{\hat{C}_{b}-\hat{C}}{d_{b}-d}
$$


where $\hat{C}$ and $d$ are the discrepancies and the degrees of freedom for the model being evaluated, and $\hat{C}_{b}$ and $d_{b}$ are the discrepancies and the degrees of freedom for the baseline model, there are another indicators such as The Tucker-Lewis coefficient $\left(\rho_{2}\right.$ in the notation of (Bollen, 1989b) ${ }^{[8]}$, and calculated is indicator as follows:

$$
T L I=\rho_{2}=\frac{\frac{\hat{C}_{b}-\hat{C}}{d_{b}-d}}{\frac{\hat{C}_{b}}{d_{b}}-1}
$$

where $\hat{C}$ and $\mathrm{d}$ are the contradiction and the degrees of freedom for the estimated model, and $\hat{C}_{b}$ and $d_{b}$ are the contradiction and the degrees of freedom for the baseline model. To verify that the model is significant we have three good indicators; RMSEA, CFI, and GFI, the parameter estimates can be tested if the fit statistics are passible, the distribution of the ratio of each estimate parameter to its standard error is t-statistic and at the 0.05 level it is significant if the value surpasses 1.96 and it is significant if the value surpasses 2.56 at level $0.01^{[16]}$, to understand how the factors related together, correlations between the latent factors should be checked

\subsection{Types of Models in SEM:}

Different types of structural equation models are used in research ${ }^{[28]}$, such as path analytic models, confirmatory factor analysis models, Structural regression models, and Latent change model. Path analytic model visualize the range of observed variables. Its remarkable part of historical expansion of SEM and use the same process of model testing and fitting as other SEM models. In addition to understand patterns of relationships between various constructs, every construct in the model is measured by a group of observed variables confirmatory factor analysis model is used. Structural regression models is another type of SEM that build on the CFA models by assuming particular explanatory relationship between constructs. SR model is constantly used to test or disconfirm suggested theories including explanatory relationships between various latent variables, to study change time the latent models are used. Such as; focus on patterns of growth, decline, or both in longitudinal data ${ }^{[26], ~[34]}$.

\subsection{Model Specification and Path Diagram:}

It is used to display confirmatory factor models as path diagrams which are use squares to represent observed variables and circles to represent the latent variables. Figure 1 has five latent variables $\alpha 1, \ldots, \alpha 5$ in circles that are manifested by eighteen observed variables in squares. The circles labeled $\alpha$ (i) represent latent variables or factors. A factor can point to more than one observed variable; in Figure 1, $\alpha 1$ causes five observed variables X1 through X5, $\alpha 2$ causes four observed variables Z1 through Z4, $\alpha 3$ causes three observed variables M1 through M3, $\alpha 4$ causes three observed variables $\mathrm{N} 1$ through $\mathrm{N} 3$, and $\alpha 5$ causes three observed variables Y1 through Y3, the covariance between $\alpha$ (i) represent $\theta \mathrm{ij}$ $(i, j=1, \ldots, 4)$ on the two-headed arrow. Factor loadings are represented by $\lambda \mathrm{ij} ;(\mathrm{i}, \mathrm{j}=1, \ldots, 18)$. The squared factor loading $\lambda_{i j}^{2}$ is referred to as a communality representing the proportion of variance in the ith observed variable that is explained by the jth latent variable ${ }^{[9]}$.

Circles named $e_{i}$ are unique factors because they have an impact on a single observed variable, in each xi the ei combine all the variance, such as measurement error, which is not holded by the common factors. 


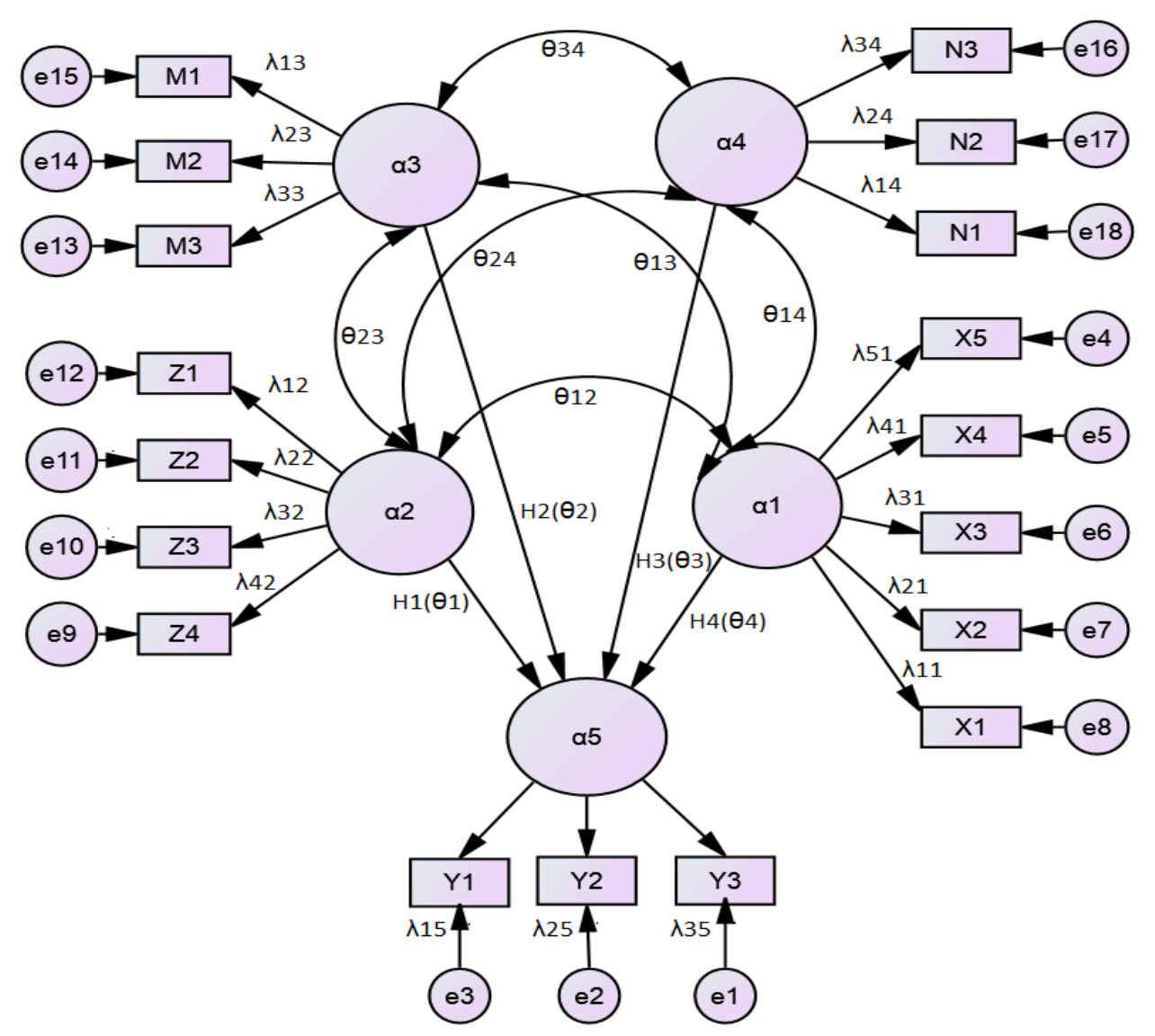

Figure (1): Research Model

The confirmatory factor model can be summarized by the equation 7, while latent, and observe variables are centered to have deviations from their means ${ }^{[34]}$ :

$$
\aleph=\Lambda \alpha+e
$$

where $\boldsymbol{N}$ is the observed variables vector, the matrix of factor loadings to connected the $\alpha \mathrm{i}$ to the $\mathrm{Xi}$ is $\Lambda$ (lambda), $\alpha$ is the vector of common factors, and ei is represented the vector of unique factors. It is supposed that the mean of the error terms is zero, $\mathrm{E}(\mathrm{ei})=0$, and there is no correlation between the common factors and unique factors, $\mathrm{E}(\alpha \mathrm{ei})=0$. Can be rewritten the equation 7 for fit the Figure 1 as ${ }^{[33],[34]}$ :

$$
X_{1}=\lambda_{11} \alpha_{1}+e_{8}, X_{2}=\lambda_{21} \alpha_{1}+e_{7}, X_{3}=\lambda_{35} \alpha_{1}+e_{6}
$$

From the equation 8 can be see the similarities with regression model. Each $\mathrm{Xi}$ is a linear function of one or more mutual factors with an error term. In CFA $\alpha$ i are hidden, and that is the major difference between factor equations and regression analysis. Thus the estimation is done in a way that is a different from the classical approach of regressing each $\mathrm{x}$ on the $\alpha \mathrm{i}^{[34]}$.

\subsection{Hypothesis Constructions:}

The study hypothesis states that the factors have a significant relationship with abortion problem and these factors also have a protective effect on the abortion issue in Sulaimani city. Accordingly, we postulate that: the first hypothesis is H1. "The previous disease information, which is represented as the indicator, is jointly significant effects on occurring 
abortion issue", so the second is a state that $\mathrm{H} 2$. "The food and drug information, which is represented as an indicator, jointly exerts influence on occurring abortion issue", and the third hypothesis is that H3. "The patient address, which is represented as the indicator, is jointly significant influence on occurring abortion issue", the final hypothesis is that $\mathrm{H} 4$. "Mother's information, which is represented as the indicator, jointly exerts effects on occurring abortion issue".

\subsection{Estimation:}

Let $\mathrm{x}$ variables are measured as deviations from their means it is easy to explain that the sample covariance matrix for $\mathrm{x}$, denoted by $\mathrm{S}$, and can be decomposed as below ${ }^{[33], ~[34]}$ :

$$
\Sigma=\Lambda \Phi \hat{\Lambda}+\delta
$$

where $\Phi$ (phi) is covariance matrix of the $\alpha$ factors, and $\delta$ (delta) is the covariance matrix of the unique factors e ${ }^{[32]}$. By obtaining the parameters $\widehat{\Lambda}$ can be estimation proceeds, the predicted covariance matrix $\Sigma$ (sigma) are $\widehat{\Phi}$, and $\hat{\delta}$ which is as close to the sample covariance matrix $\mathrm{S}$. To determining the nearest volume of covariance matrix to the sample covariance matrix there are various fitting functions, such as maximum likelihood which is the most popular. The variances, regression parameters, and covariance between variables are parameters of a SEM, while the coefficients of regression is appear as a single-headed arrows which is shows pathway of hypothesis between two variables, however the covariance is appear as double-headed, curved arrows between two variables ${ }^{[18]}$.

\section{Data Analysis and Results}

\subsection{Data Description:}

Data were obtained by a survey from a Maternity hospital in Sulaimani, Kurdistan-Iraq. We used these data because the women's patient remains at this hospital. In addition, the Maternity hospital is number one area for pregnant women to obtain information about the abortion issue. Therefore, we could communicate with these patients such activities to patients ${ }^{[31]}$. One hundred and twenty questionnaires were distributed to the patient, ninety-six questionnaires were received, and the questionnaires were prepared in Kurdish language and then translated into English. The variables were utilized in this study as follows: food and drug information, patient address, abortion information, which are consisted of three items, while mother's information consisted of five items, and previous disease information consisted of four items.

\subsection{Research hypothesis check:}

To estimate the structural equation models parameters can be use maximum likelihood estimation method ${ }^{[1]}$, the results are shown in Table 2 and Figure (2); which are explain that the X1 appear to be most reliable indicator from mother's information factor by amount $\left(\lambda_{11}=0.76\right)$, and then X4, X5, X3, and X2 after X1 of the same factor by amounts $\left(\lambda_{41}=0.28, \lambda_{51}=0.21, \lambda_{31}=-0.03\right.$, and $\left.\lambda_{21}=-0.03\right)$ respectively, while in the (previous disease information) factor, Z4 appear a high indicator by amount $\left(\lambda_{42}=0.84\right)$, after that Z3, Z2, and Z1 are most dependable measurements after $Z 4$ by amounts $\left(\lambda_{32}=-0.73, \lambda_{22}=0.44\right.$, and $\left.\lambda_{12}=-0.24\right)$ respectively, although $\mathrm{M} 2$ seems to be most credible measurement in the factor food and drug information by amount $\left(\lambda_{23}=0.32\right)$, then M1 and M3 appear to be most reliable indicators after M2 in the same factor by amount $\left(\lambda_{13}=0.25\right.$, and $\left.\lambda_{33}=0.21\right)$ respectively, in the patient address factor, $\mathrm{N} 1$ seems to be most dependable measure by amount $\left(\lambda_{14}=-0.44\right)$, also N3 and N2 followed N1 by amounts $\left(\lambda_{34}=0.41\right.$, and $\left.\lambda_{24}=0.24\right)$ respectively, Y1 appear to be most reliable indicator in the last factor (abortion information) by amount $\left(\lambda_{15}=-0.84\right)$, then $\mathrm{Y} 2$, and $\mathrm{Y} 3$ by amounts $\left(\lambda_{25}=0.78\right.$, and $\lambda_{35}=0.09$ ) respectively. The empirical data are supported the study hypothesizes because food and drug information, patient address, mother's information, and previous disease information are exerted an impact on occurring abortion by amounts $\left(\theta_{3}=\right.$ 
$-1.28, \theta_{4}=0.75, \theta_{1}=0.72, \theta_{2}=0.02$ ) respectively ${ }^{[4,13,15]}$. In addition the results explain $70 \%, 61 \%, 1 \%$ of the variance in occurring abortion issue according to Y1, Y2, and Y3 respectively as shown in Table $2^{[24]}$.

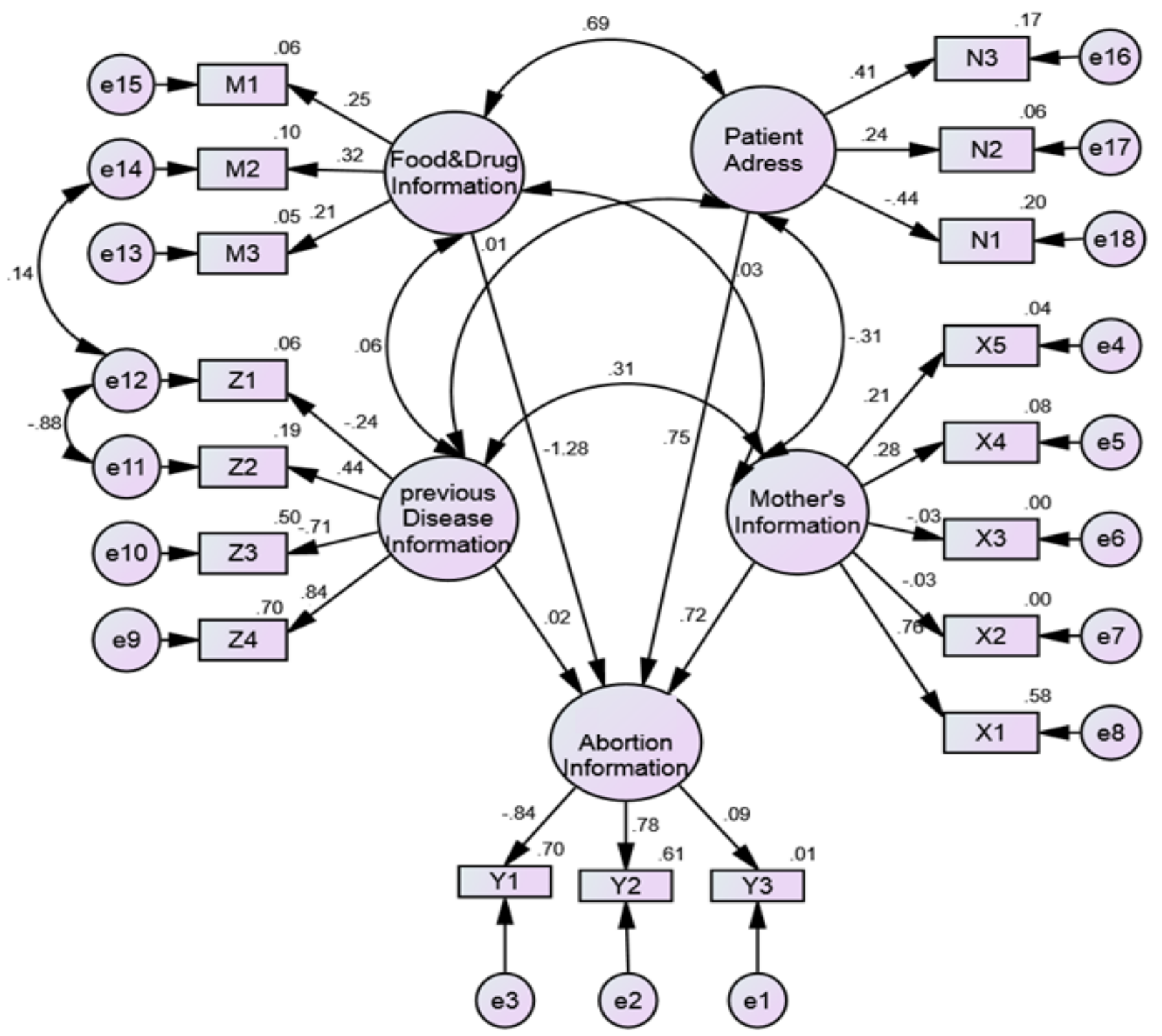

Figure (2): Modify SEM results for Abortion Factors 
From figure (2), can be explain all symbols with meaning as in the following table (1):

Table (1): Variables code with meaning

\begin{tabular}{ccl}
\hline NO. & Symbols & \\
\hline 1 & X1 & Patient age \\
2 & X2 & Occupation \\
3 & X3 & Patient blood group \\
4 & X4 & Are patient and her partner cousins \\
5 & X5 & Use pregnancy control pills prior to her pregnancy \\
6 & N1 & Patient address \\
7 & N2 & Are any of building type (manufacture, and electronic tower) close to patient \\
& & living area \\
8 & N3 & which of these (internet, and cell phone) did patient use the most during \\
& & pregnancy \\
9 & M1 & Drink any of (Dates, Animal liver, and Honey) before becoming pregnant \\
10 & M2 & Use any medication during pregnancy \\
11 & M3 & Use any of (Aspirin, and Cervical process) to prevent abortion \\
12 & Z1 & Have any prior disease \\
13 & Z2 & Type of prior disease \\
14 & Z3 & Have any illness during her pregnancy \\
15 & Z4 & Type of illness during pregnancy \\
16 & Y1 & Previous abortion of Patient \\
17 & Y2 & Number of abort child \\
18 & Y3 & Age of abort child \\
\hline
\end{tabular}

\subsection{Measurement model check:}

Table 2 shows the variables dropped as a result of confirmatory factor analysis. The overall model fit supported the measurement model $\left(\chi^{2}=124.45, \mathrm{df}=124, \mathrm{p}-\right.$ value $=.472, \chi^{2} / \mathrm{df}=1.004, \mathrm{RMSEA}=.006, \mathrm{GFI}=.880, \mathrm{TLI}=$ $.998, \mathrm{CFI}=.999, \mathrm{IFI}=.999)$. As reported in Table 2 , note that the $\chi^{2}$ statistic for model fit is not significant, meaning that the null hypothesis of a good fit to the data can't be rejected. The RMSEA likewise suggests that the fit of the model is an achievement, which is less than cut-off value for accepting the model fit that is equal to $0.05^{[31]}$, statistics and factor loading and square multiple correlation of observed variables are given in Table 2 and model fit statistics measurements in Table 3:.

Table (2): Factor Loading and Square Multiple Correlations (SMC) Results 


\begin{tabular}{lccc}
\hline Scale Variables & $\begin{array}{c}\text { Unstandardized Loading } \\
\text { (Standard Error) }\end{array}$ & $\begin{array}{c}\text { Standardized } \\
\text { Loading }\end{array}$ & $\begin{array}{c}\text { Square Multiple } \\
\text { Correlation (SMC) }\end{array}$ \\
\hline mother's information & $37.282(37.8)$ & 0.761 & 0.58 \\
X1 & $-0.242(.98)$ & -0.033 & 0.001 \\
X2 & $-0.52(2.55)$ & -0.027 & 0.001 \\
X3 & $1.321(1.01)$ & 0.28 & 0.078 \\
X4 & 1 & 0.21 & 0.044 \\
X5 & & & \\
previous disease information & $-0.02(.008)$ & -0.244 & 0.06 \\
Z1 & $0.224(.057)$ & 0.435 & 0.19 \\
Z2 & $-0.071(.013)$ & -0.708 & 0.501 \\
Z3 & 1 & 0.84 & 0.70 \\
Z4 & & & \\
food and drug information & $3.316(2.84)$ & 0.246 & 0.06 \\
M1 & $0.78(.655)$ & 0.319 & 0.101 \\
M2 & 1 & 0.215 & 0.046 \\
M3 & & & \\
patient address & $-0.717(.524)$ & -0.444 & 0.197 \\
N1 & $0.496(.416)$ & 0.244 & 0.06 \\
N2 & 1 & 0.407 & 0.165 \\
N3 & & & 0.705 \\
previous abortion information & $-0.654(.788)$ & -0.839 & 0.609 \\
Y1 & $1.613(2.007)$ & 0.78 & 0.009 \\
Y2 & 1 & 0.095 & \\
Y3 & & & \\
\hline
\end{tabular}

Table (3): Model fit statistics measurements:

\begin{tabular}{lll}
\hline \multicolumn{1}{c}{ Measurement } & \multicolumn{1}{c}{ Values } & meaning \\
\hline$\chi^{2}$ & 124.45 & Chai-square test \\
$\mathrm{df}$ & 124 & Degree of freedom \\
$\mathrm{p}-$ value & 0.472 & Probability (significant) value \\
$\chi^{2} / \mathrm{df}$ & 1.004 & Chai-square over degree of freedom \\
RMSEA & 0.006 & Root mean square error of approximation \\
GFI & 0.880 & Goodness fit index \\
TLI & 0.998 & Tucker-Lewis index \\
CFI & 0.999 & Comparative fit index \\
IFI & 0.999 & Incremental-fit index \\
\hline
\end{tabular}

Parameter estimates for the re-specified model are presented in Table 2, the variances are in standard deviation form, the inter-factor associations are expressed as correlations, and the factor loadings reflect the number of standard units change in the indicators per standard unit change in the factor(s) on which they load. Reflecting the re-specification of the five-factor model, in the final column of Table 2 are MSC values for each item, these values indicated the proportion of variance in each item attributable to the factor(s) on which it loads, that contributed to variability in the responses ${ }^{[7]}$. 
Table (4): Summary structural equation results.

\begin{tabular}{|c|c|c|c|c|c|c|}
\hline Effect & Scale Variables & & $\begin{array}{l}\text { mother's } \\
\text { informatio } \\
\mathrm{n}\end{array}$ & $\begin{array}{l}\text { previous } \\
\text { disease } \\
\text { information }\end{array}$ & $\begin{array}{l}\text { food and } \\
\text { drug } \\
\text { information }\end{array}$ & $\begin{array}{l}\text { patient } \\
\text { address }\end{array}$ \\
\hline $\begin{array}{l}\text { Direct } \\
\text { Effect }\end{array}$ & $\begin{array}{l}\text { Previous } \\
\text { Information }\end{array}$ & \multirow[t]{2}{*}{ Abortion } & 0.719 & 0.022 & -1.279 & 0.752 \\
\hline $\begin{array}{l}\text { Indirect } \\
\text { Effect }\end{array}$ & $\begin{array}{l}\text { Y1 } \\
\text { Y2 } \\
\text { Y3 }\end{array}$ & & $\begin{array}{l}-0.603 \\
0.561 \\
0.068\end{array}$ & $\begin{array}{l}-0.018 \\
0.017 \\
0.002\end{array}$ & $\begin{array}{l}1.074 \\
-0.999 \\
-0.121\end{array}$ & $\begin{array}{l}-0.632 \\
0.587 \\
0.071\end{array}$ \\
\hline Total & $\begin{array}{l}\text { Previous } \\
\text { Information }\end{array}$ & \multirow[t]{4}{*}{ Abortion } & 0.719 & 0.022 & -1.279 & 0.752 \\
\hline Effect & Y1 & & -0.603 & -0.018 & 1.074 & -0.632 \\
\hline & $\mathrm{Y} 2$ & & 0.561 & 0.017 & -0.999 & 0.587 \\
\hline & Y3 & & 0.068 & 0.002 & -0.121 & 0.071 \\
\hline
\end{tabular}

Table 4. shown direct, indirect and total effects of all factors on occurring abortion, the direct effect of each factors (mother's information, previous disease information, and patient address on abortion issue) are $(0.719,0.022,0.752)$ respectively while the indirect effect of the same factors are ([-0.60, 0.56, 0.07], $[-0.02,0.02,0.002]$, and $[-0.63,0.59$, $0.07])$ on (y1, y2, and $y 3)$ for each factors above respectively ${ }^{[14]}$. But food and drug information factor have a negative effect on occurring abortion the amount of this effect is (-1.28) and the indirect effect of the same factor are (1.07, -0.99 , and -0.12$)$ on $(\mathrm{y} 1, \mathrm{y} 2$, and $\mathrm{y} 3)$ respectively ${ }^{[12]}$.

\section{Results and Conclusions:}

There are three implications emerging from the findings of this study. First, there investigated the consequences of factors that caused occurring abortion issue. It's an important factor because the overwhelming majority of the empirical studies have assessed these factors based on simple statistical mechanism. By doing so, our study attempts to fill the void in the healthy field of general pregnancy problems. The effect of patient address factor on abortion problem the one. Beside the positive direct effect of this factor, more difference patient area (address) such as; (living in urban, rural, and existing building type ( manufacture or electronic tower) close to the patient area) leads to more occurring abortion because of differencing in area lead to differencing in healthy culture, then the effect of mother information (like; age, occupation, blood group, existing patient and her partner cousins, and use pregnancy control pills prior to patient pregnancy have strong positive effect on abortion issue, it may be concluded that this result concerning the effects of this factor come from the fact that each elements in this factor have an effect on abortion, in case of increasing in age and hard work lead to increasing risk on occurring abortion, emphasize this result by Ahmed et al. (1996) ${ }^{[3]}$. While the impact of food and drug information factor such as; before becoming pregnant drink any of item (Dates, Animal liver, and Honey), or taking any medication during pregnancy like (Aspirin, and cervical process) is negative, it may be concluded that this result concerning the direct effect of this factor comes from the fact that the less food and medication during (without advice doctor) lead to increasing risk on occurring abortion issue. Also the previous disease factor had a less effect on abortion problem, because of the mother beware her healthy when she has previous disease.

\section{References:}

A.M. Kwon, C. Shin, (2016), "structural equation modeling for the effect of physical exercise on excessive daytime sleepiness", journal of public health, The Royal Society for Public Health. Published by Elsevier Ltd. 
A.S. Goldberger and O.D. Duncan (Ed.), (1973), "Structural Equation Models in the Social Sciences", (pp. 85-112), Semin ar Press, New 430 York.

Ahmed shameem, Haque Indrani, Barkat-e-Khuda, Hossain Bazle Main, Alam Shahidul, (1996), "Abortion in Rural Bangladesh", Evidence from the MCH-FP Extension Project (Rural), Health and population extension division, ICDDR,B center for health and population research.

Bagozzi, R.P., Yi, Y., (1988), "On the evaluation of structural equation models", J. Acad. Mark. Sci. 16 (1), 74-94. Bartlett LA et al., (2004) "Risk factors for legal induced abortion-related mortality in the United States", Obstetrics and Gynecology, 103:729-737.

Bentler, P. M., \& Bonett, D. G., (1980), "Significance tests and goodness-of-fit in the analysis of covariance structures", Psychological Bulletin, 88, 588-600.

Bollen, K.A. (1989), “Structural Equation Models with Latent Variables”, New York: Wiley \& Sons.

Bollen, K.A. (1989b), "Anew incremental fit index for general structural equation models", Sociological Methods and Research, 17, 303-316.

Brown, T. A., (2006), “Confirmatory Factor Analysis for Applied Research”, New York: Guilford Press.

Byrne, B. M., (2006), "Structural equation modeling with EQS": Basic concepts, applications, and programming (2 $2^{\text {nd }}$ ed.). Mahwah, NJ: Erlbaum.

Child, D., (1990), “The essentials of factor analysis", ( $2^{\text {nd }}$ ed.). London, England: Cassel Academic.

Eshima Nobuoki, Tabata Minoru, Zhi Geng, (2001), "Path Analysis with Logistic Regression Models": Effect Analysis of Fully Recursive Causal of System of Categorical Variables, J. Japan Statist. Soc., Vol. 31, No. 1.

Fornell, C., Larcker, D.F., (1981), "Evaluating structural equation models with unobservable variables and measurement error", J. Mark. Res. 18 (1), 39-50.

Grossman M. (1975), “The Correlation Between Health and Schooling”, In Terleckyj N. (eds.) Household Production and Consumption. Columbia University Press, New York.

Hair, J.F., Black, W.C., Babin, B.J. and Anderson, R.E., (2010), “Multivariate data analysis", a global perspective $7^{\text {th }}$ ed., New Jersey: Pearson Prentice Hall.

Hoyle, R. H. (1995), “The structural equation modeling approach": Basic concepts and fundamental issues. In R. H. Hoyle (Ed.), Structural equation modeling: Concepts, issues, and applications (pp. 1-15).Thousand Oaks, CA: Sage Publications.

Hoyle, R. H. (Ed.), (2012), “Handbook of structural equation modeling”, New York: Guilford Press. Hoyle, R.H. (ed.), (1995), "Structural Equation Modeling", SAGE Publications, Inc. Thousand Oaks, CA. This book provides a very readable, broken-down introduction to SEM. It discusses SEM in relation to AMOS software.

Hu, L. \& Bentler, P. M., (1999), "Cutoff criteria for fit indexes in covariance structure analysis": Conventional criteria versus new alternatives. Structural Equation Modeling, 6, 1-55. 
Jiang Wen-Xiao, He Fang-Fang, Shen Qi, Tao Xue-Jiao, Zhao Chu-Chu, Shen Zhao-Jun, Zhu Xue-Qiong, (2017), "Factors related to completeness of medical abortion with mifepristone and misoprostol", original article, Journal of the Chinese Medical Association 80, 96-102.

Jöreskog, K.G. \& Sörbom, D., (1984), “LISREL-VI user's guide”, 3d ed., Mooresville, IN: Scientific Software. Jöreskog, K.G. (1969), “A general approach to confirmatory factor analysis", Psychometrika, 34, 183-202. K.A. Bollen and J.S. Long (Ed.), (1993), "Testing Structural Equation Models", (pp. 163-180), Beverly Hills, CA: Sage.

Koufteros X.A., (1999), “Testing a model of pull production: a paradigm for manufacturing research using structural equation modeling", Journal of Operations Management, 17, 4, 467-488.

Leonel Briozzo, (2016), "From risk and harm reduction to decriminalizing abortion: The Uruguayan model for women's rights", International Journal of Gynecology \& Obstetrics, Volume 134, Supplement 1, Pages S3-S6.

Mamboleo Neema, (2012), "Unwanted Pregnancy and Induced Abortion Among Female Youths: A case study of Temeke District", Master of Public Health Dissertation, Muhimbili University of Health and Allied Sciences.

Oliver-Williams T. Clare, Steer J. Philip, (2015), "Racial variation in the number of spontaneous abortions before a first successful pregnancy, and effects on subsequent pregnancies", CLINICAL ARTICLE, International Journal of Gynecology and Obstetrics 129, 207-212.

Raykov, T., \& Marcoulides, G. A., (2006), “A first course in structural equation modeling”, $2^{\text {nd }}$ ed., Mahwah, NJ: Erlbaum.

Sedgh G, et al. (2012), "Induced abortion: incidence and trends worldwide from 1995 to 2008". Lancet, 379:625632 .

Singh S et al. (2009), "Abortion worldwide: a decade of uneven progress", New York, Guttmacher Institute. Steiger, J. H. and Lind, J. (1980), "Statistically-based tests for the number of common factors", Paper presented at the Annual Spring Meeting of the Psychometric Society, Iowa City.

Vlassoff $\mathrm{M}$ et al. (2008), "Economic impact of unsafe abortion-related morbidity and mortality: evidence and estimation challenges", Brighton, Institute of Development Studies, (IDS Research Reports 59).

X.Y. Song, S.Y. Lee, (2012), "Basic and Advanced Bayesian Structural Equation Modeling: With Applications in the Medical and Behavioral Sciences", 455 Wiley, Chichester.

Yan-Qing Zhang , Guo-Liang Tian , Nian-Sheng Tang , (2016), "Latent variable selection in structural equation models", Journal of Multivariate Analysis. http://www.who.int/csr/resources/publications/EPR_AM2_E7.pdf. 